\title{
Clinical and electrophysiological recovery in Leber hereditary optic neuropathy with G3460A mutation
}

\author{
Eamon Sharkawi · Justyna D. Oleszczuk • \\ Graham E. Holder · Joyti Raina
}

Received: 27 March 2012/Accepted: 20 April 2012/Published online: 10 June 2012

(C) Springer-Verlag 2012

\begin{abstract}
To report a case of clinical and electrophysiological recovery in Leber hereditary optic neuropathy (LHON) with G3460A Mutation. A 10 -year-old boy with a three-month history of painless bilateral sequential visual loss upon presentation underwent visual acuity (diminished), anterior and posterior segment examination (normal), fluorescein angiography (normal), Goldman kinetic perimetry (bilateral central scotomata), genetic (a point G3460A mutation) and electrophysiological investigation (undetectable pattern visual evoked potentials (VEP); low amplitude, broadened and reduced flash VEPs and loss of the N95 component in the pattern electroretinograms). Diagnosis of LHON was made. Eighteen months later vision and electrophysiological tests results began spontaneously improving. Kinetic
\end{abstract}

E. Sharkawi $(\bowtie) \cdot$ J. D. Oleszczuk

Jules-Gonin Eye Hospital, Avenue de France 15,

Lausanne 1004, Switzerland

e-mail: eamon.sharkawi@fa2.ch

\section{G. E. Holder}

Department of Electrophysiology, Moorfields Eye

Hospital, London ECV1 2PD, UK

\section{G. E. Holder}

Institute of Ophthalmology, University College London, London, UK

J. Raina

Department of Ophthalmology, North Middlesex

University Hospital, London N18 1QX, UK perimetry revealed reduced density and size of scotomata. Two years later, there had been further electrophysiological improvement. This report describes both clinical and electrophysiological improvement in LHON with G3460A mutation.

Keywords Leber hereditary optic neuropathy · LHON · Electrophysiology · VEP · G3460A ·

Recovery

\section{Introduction}

Leber hereditary optic neuropathy (LHON) is a rare, mitochondrially inherited disorder usually characterized by sequential bilateral visual loss, severe optic nerve dysfunction and a poor prognosis. The present report describes a case of late visual recovery and improvement in electrophysiological function in a child with the G3460A mutation.

\section{Case report}

A 10-year-old boy presented with a three-month history of painless bilateral sequential visual loss. He was unable to recognize faces, read or watch television, although he managed to navigate around his home and school. Visual acuities (VA) were hand movements in the right eye (OD), counting fingers in the left eye (OS). Anterior and posterior segment 
examinations, including optic disc appearances, were normal. Pupillary reflexes were sluggish. Fluorescein angiography was unremarkable. Goldman kinetic perimetry revealed bilateral fenestrated central scotomata with islands of varying density (Fig. 1a, b). Electrophysiological investigation (Fig. 2a) showed undetectable pattern VEP (PVEP); low amplitude, broadened and reduced flash VEPs (FVEP) and loss of the N95 component in the pattern electroretinograms (PERG). Full field ERGs (not shown) were normal. Mitochondrial DNA testing revealed a point mutation,
G3460A, previously associated with LHON. He was registered blind, plans were made for special schooling and the family was offered genetic counselling.

Eighteen months later, his vision began spontaneously improving and by 24 months, VA was $6 / 18$ right, 6/12 left, associated with reduced disability. Electrophysiological investigation showed improved amplitude in PVE and FVEP and some improvement in the N95:P50 ratio of the $15^{\circ}$ PERG (Fig. 2b). Kinetic perimetry revealed reduced density and size of scotomata (Fig. $1 \mathrm{c}, \mathrm{d}$ ).

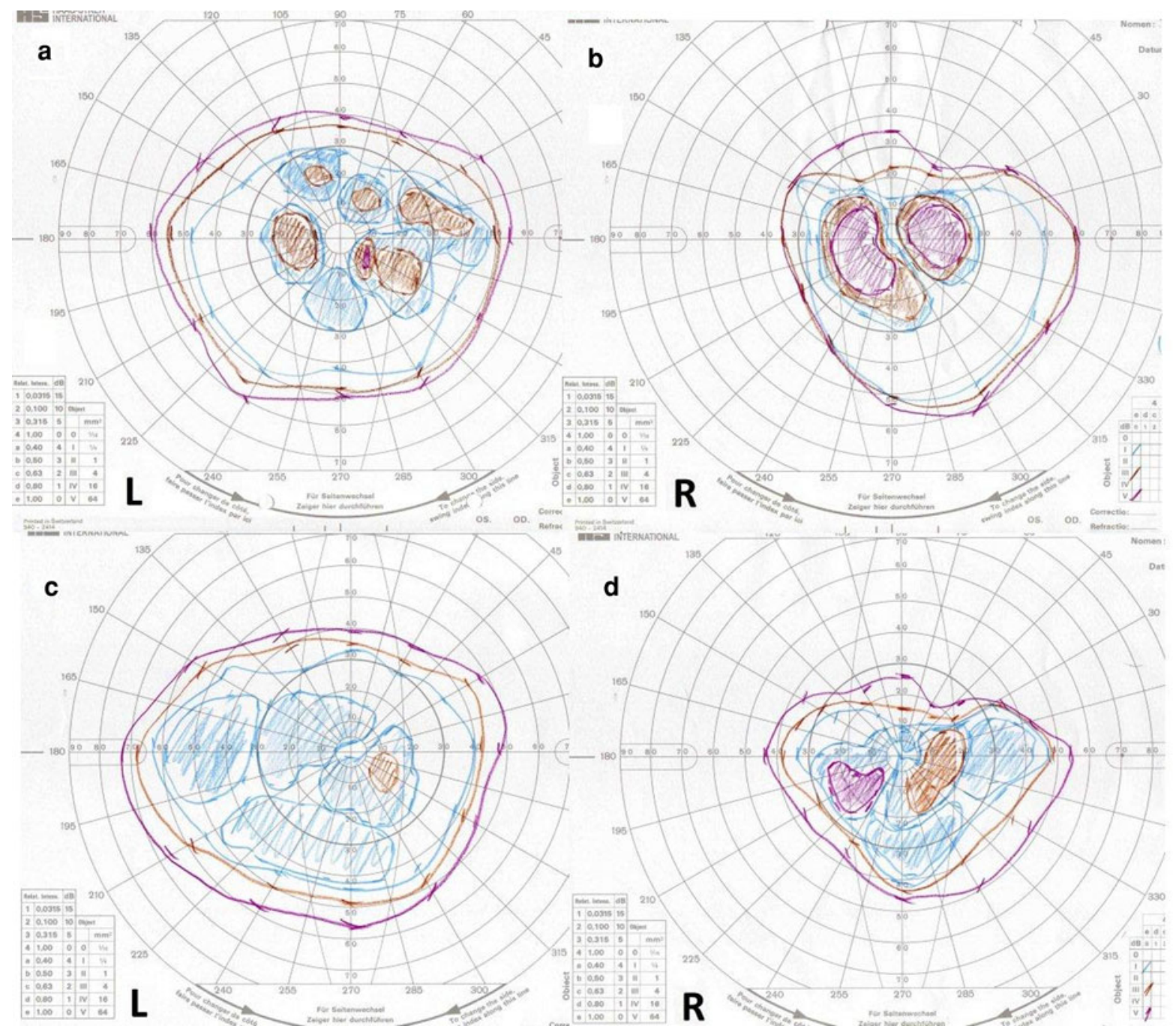

Fig. 1 Left and right Goldman visual fields at presentation show bilateral central fenestrated scotomata (a, b); 24 months later, there is reduction in the density and size of the scotomata $(\mathbf{c}, \mathbf{d})$ 
Fig. 2 a July 2003. VAR hand movements; VAL counting fingers. PVEP, FVEP, $15^{\circ}$ PERG and $30^{\circ}$ PERG. PVEP is undetectable bilaterally. FVEPs are of low amplitude, broadened and reduced; there is loss of the N95 component in the $15^{\circ} \mathrm{PERG}$ and reduction in the $30^{\circ}$ PERG. b October 2005.

VAR 6/18; VAL 6/12.

PVEP is now detectable, but delayed; FVEP shows recovery in amplitude; $15^{\circ}$ PERG N95 component shows some recovery, but remains markedly abnormal; $30^{\circ}$ PERG was not performed. c October 2007. VAR 6/18; VAL 6/12. RE PVEP is of low amplitude but now normal peak time; left eye PVEP remains delayed but is improved; FVEPs are normal; PERGs show no evident change a
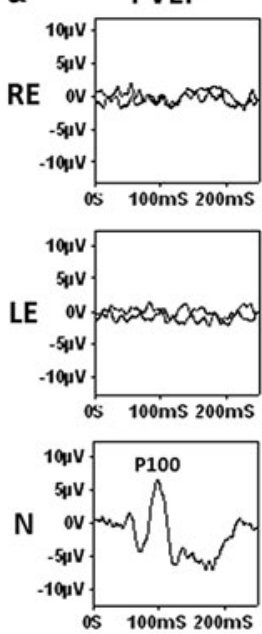

b
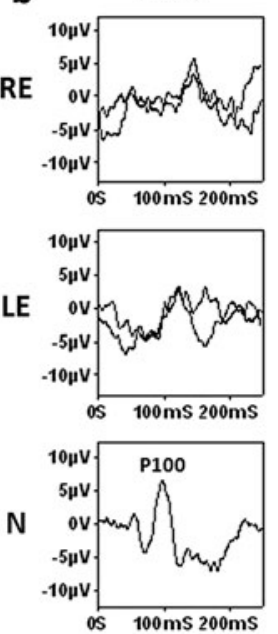

C

PVEP
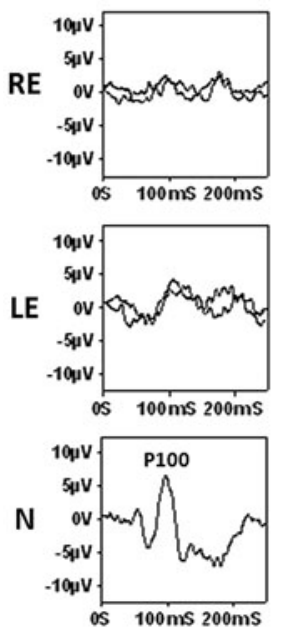
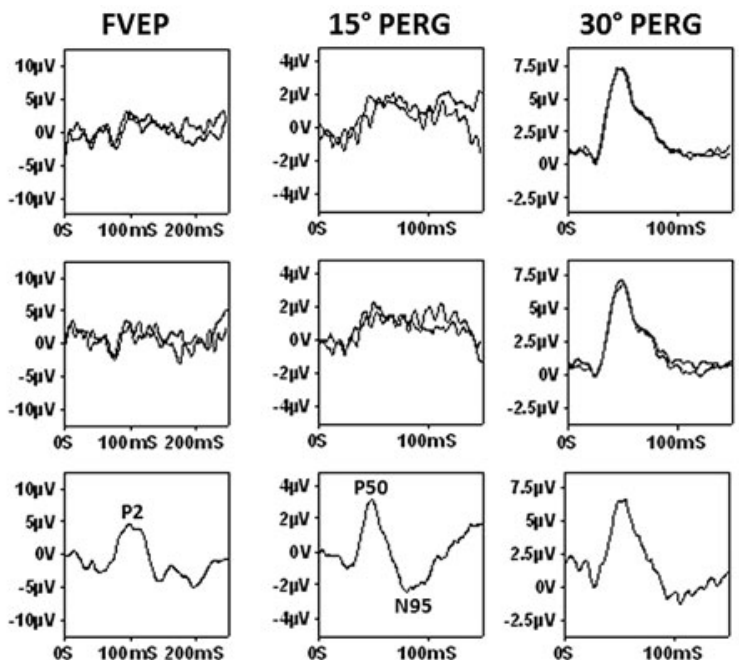

FVEP

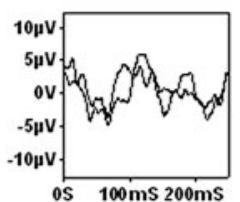

$15^{\circ}$ PERG
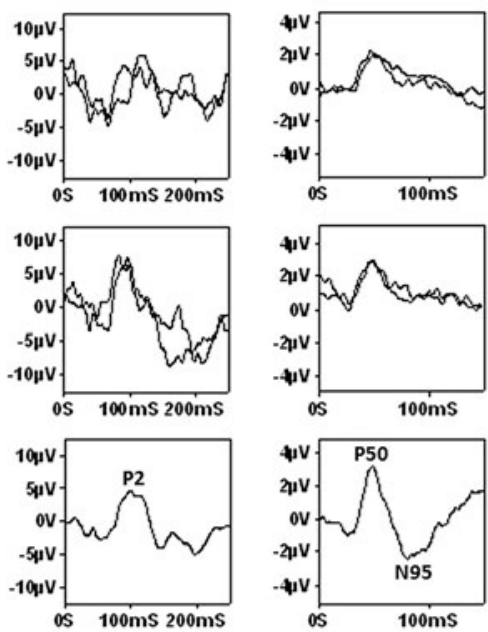

FVEP
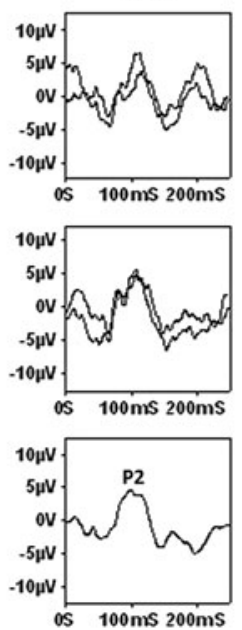

$15^{\circ}$ PERG
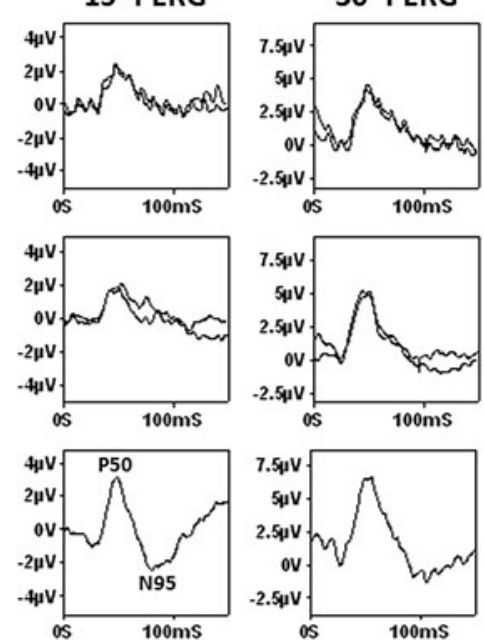
Two years later, there had been further electrophysiological improvement (Fig. 2c). The patient remained clinically stable 4 years after initial presentation without the need for special schooling.

\section{Discussion}

This report describes a case of molecularly confirmed G3460A mutation Leber Hereditary Optic Neuropathy in which there was clinical improvement commencing approximately 18 months after the onset of visual loss. This was accompanied by electrophysiological improvement, which to our knowledge has not been previously reported.

Leber hereditary optic neuropathy is the commonest of the mitochondrial diseases and passes through the maternal line [1-3]. Approximately, $95 \%$ of LHON pedigrees have one of three mitochondrial DNA point mutations, G3460A, G11778A and T14484C, which are involved in encoding complex I subunits of the mitochondrial respiratory chain [1, 2, 4-7]. These mutations account for approximately 15,70 and $10 \%$ of LHON pedigrees, respectively [5-7]. Heteroplasmy, secondary genomic and mitochondrial factors and environmental triggers are thought to contribute to disease expression [4, 5, 8]. Clinical recovery in LHON can occur spontaneously several months to years after initial presentation and is least likely with the 11778 mutation (approximately $5 \%$ of patients), followed by $22 \%$ in the 3460 mutation and up to $65 \%$ in the 14484 variant (reported recovery rates vary from 37 to $65 \%)[1,6,7]$. The mechanism of spontaneous delayed visual recovery remains unknown, but it has been suggested that a subset of inactive, but viable retinal ganglions cells (possibly homoplasmic for the wild allele), may be responsible [5, 9].

Barboni et al. [10] reported childhood-onset disease to follow a milder course (especially in slowly progressive cases) and to have higher recovery rates than in affected adults. The development and enlargement of small vision islands within central scotomata was reported to be typical in paediatric patients [9]. There is a lack of published data regarding electrophysiology in LHON patients following visual recovery.

The present report describes both clinical and electrophysiological improvement. Although visual recovery has been reported in the 14484 mutation, one report with VEP did not find improvement in PVEP [3]. Flash VEPs in that patient were normal. Although it has been suggested that luminance-related fibres may be less affected than contrast-related fibres in patients with early LHON [11], flash VEPs are generally less sensitive to disease than pattern VEPs. Further detailed electrophysiological investigation of patients exhibiting visual improvement may provide additional insight into the mechanisms underlying the visual loss and delayed recovery, currently speculative.

Conflict of interests The authors declare no financial support or conflicts of interests.

\section{References}

1. Abu-Amero KK (2011) Leber's hereditary optic neuropathy: the mitochondrial connection revisited. Middle East Afr J Ophthalmol 18(1):17-23

2. Sadun AA, La Morgia C, Carelli V (2011) Leber's hereditary optic neuropathy. Curr Treat Options Neurol 13(1): 109-117

3. Hrynchak PK, Spafford MM (1994) Visual recovery in a patient with Leber hereditary optic neuropathy and the 14484 mutation. Optom Vis Sci 71:604-612

4. Kirches E (2011) LHON: mitochondrial mutations and more. Curr Genomics 12(1):44-54

5. Black GC, Morten K, Laborde A, Poulton J (1996) Leber's hereditary optic neuropathy: heteroplasmy is likely to be significant in the expression of LHON in families with the 3460 ND1 mutation. Br J Ophthalmol 80:915-917

6. Pagon RA, Bird TD, Dolan CR et al. (1993) GeneReviews $^{\mathrm{TM}}$ [online] Chapter: Leber hereditary optic neuropathy. University of Washington, Seattle WA (Last Update: July 7, 2011)

7. MITOMAP: A human mitochondrial genome database [online]. http://www.mitomap.org/MITOMAP/Mutations LHON

8. Pegoraro E, Carelli V, Zeviani M, Cortelli P, Montagna P, Barboni P, Angelini C, Hoffman EP (1996) X-inactivation patterns in female Leber's hereditary optic neuropathy patients do not support a strong X-linked determinant. Am J Med Genet 61:356-362

9. Acaroğlu G, Kansu T, Doğulu CF (2001) Visual recovery patterns in children with Leber's hereditary optic neuropathy. Int Ophthalmol 24:349-355

10. Barboni P, Savini G, Valentino ML, La Morgia C, Bellusci C, De Negri AM, Sadun F, Carta A, Carbonelli M, Sadun AA, Carelli V (2006) Leber's hereditary optic neuropathy with childhood onset. Invest Ophthalmol Vis Sci 47: 5303-5309

11. Mashima Y, Imamura Y, Oguchi Y (1997) Dissociation of damage to spatial and luminance channels in early Leber's hereditary optic neuropathy manifested by the visual evoked potential. Eye 11:707-772 